Validating the collision-dominated Child-Langmuir law for a dc discharge cathode sheath in an undergraduate laboratory

This article has been downloaded from IOPscience. Please scroll down to see the full text article.

2009 Eur. J. Phys. 301345

(http://iopscience.iop.org/0143-0807/30/6/012)

The Table of Contents and more related content is available

Download details:

IP Address: 80.92.224.98

The article was downloaded on 15/09/2009 at 10:53

Please note that terms and conditions apply. 


\title{
Validating the collision-dominated Child-Langmuir law for a dc discharge cathode sheath in an undergraduate laboratory
}

\author{
V Lisovskiy and V Yegorenkov \\ Kharkov National University, Svobody Sq. 4, 61077, Kharkov, Ukraine \\ E-mail: lisovskiy@yahoo.com
}

Received 23 June 2009, in final form 20 July 2009

Published 14 September 2009

Online at stacks.iop.org/EJP/30/1345

\begin{abstract}
In this paper, we propose a simple method of observing the collision-dominated Child-Langmuir law in the course of an undergraduate laboratory work devoted to studying the properties of gas discharges. To this end we employ the dc gas discharge whose properties are studied in sufficient detail. The undergraduate laboratory work itself is reduced to registering the voltage drop across the electrodes, the discharge current as well as the cathode sheath thickness. We can easily perform the measurements of all three quantities with sufficient accuracy in a laboratory equipped with vacuum pumps.
\end{abstract}

(Some figures in this article are in colour only in the electronic version)

\section{Introduction}

Low-temperature plasma is widely applied in a number of technological processes of etching and modifying various materials, depositing thin films, plasma cleaning technological discharge chambers, sterilizing medical tools, manufacturing plasma display panels, etc. Therefore, students in many universities study plasma physics. The plasma is created inside a discharge chamber possessing electrodes, grids, Langmuir probes, etc. A sheath forms usually near the surface of any object in contact with plasma (near a wall or an electrode). Actually the Child-Langmuir law was established in the course of studying the passage of the electric current through a vacuum tube equipped with a passive anode and a heated cathode emitting electrons, and a conventional laboratory observation is concerned with limitations posed by the electron space charge on the electron motion inside a vacuum tube [1-5]. But already Langmuir [6] pointed out the role of ions in the passage of current through gas discharge plasma and established a law relating the sheath characteristics (voltage drop across the sheath 
and sheath thickness) and plasma parameters (ion flow out of plasma at the sheath boundary) which is the most important law of plasma physics.

In its simplest form the interaction of plasma with a wall absorbing charged particles may be described as follows. Ions and electrons approaching the wall surface recombine on it and are lost from the discharge volume. Electrons possess higher thermal velocities than ions and approach the wall surface faster; therefore the plasma region near the wall is depleted of electrons. Due to higher electron mobility a wall potential happens to be negative with respect to the surrounding plasma. Electron reflection off the wall builds up a region with a positive spatial charge (sheath) screening the neutral plasma from the negative wall. The sheath extension usually amounts to several Debye scale lengths $\lambda_{D}$. The sheath also builds up near the electrodes to which a potential from the voltage source is fed.

Ions may cross the sheath without collisions with gas molecules (at pressures below 0.003 Torr for argon), the sheath being called collision-free in this case. Then the current density of ions $J$ is related to the voltage drop across the sheath $U$ and the sheath thickness $d$ via the Child-Langmuir law [6-8]:

$$
J=K_{i} \varepsilon_{0}\left(\frac{2 e}{M}\right)^{1 / 2} \frac{U^{3 / 2}}{d^{2}},
$$

where $K_{i}=200 / 243=0.82$ [8], $\varepsilon_{0}$ is the vacuum permittivity constant, $e$ is the elementary charge and $M$ is the ion mass. In the law (1) the ion current density $J$ is proportional to the voltage drop across the sheath $U$ raised to the power of $3 / 2$; therefore, the Child-Langmuir law is often referred to as the law of ' $3 / 2$ '.

The collision-free Child-Langmuir law (1) fails to describe the motion of ions across the sheath when they collide underway with gas molecules. In this case one has to employ the following modified Child-Langmuir law taking into account the limited ion mean free path $\lambda_{i}$ [8]:

$$
J=1.68 \varepsilon_{0}\left(\frac{2 e \lambda_{i}}{M}\right)^{1 / 2} \frac{U^{3 / 2}}{d^{5 / 2}} .
$$

The ion mean free path $\lambda_{i}$ is inversely proportional to the gas pressure $p$. For example, for argon we have $\lambda_{i}=1 /(330 p) \mathrm{cm}$, where the gas pressure $p$ is in Torr. Formula (2) can be applied when the parameter $\lambda_{i}$ is independent of ion velocity.

When the ion mobility $\mu_{i}$ is independent of velocity, the collision-dominated ChildLangmuir law assumes the form [8]

$$
J=\frac{9}{8} \varepsilon_{0} \mu_{i} \frac{U^{2}}{d^{3}} .
$$

Generally formulae (1)-(3) can be written as follows:

$$
J=k \frac{U^{m}}{d^{n}}
$$

where for (1) $m=1.5$ and $n=2$, for (2) $m=1.5$ and $n=2.5$, and for (3) $m=2$ and $n=3$.

A dc glow discharge appears under avalanche gas breakdown and it possesses a large voltage drop across the cathode sheath. The glow discharge consists of the following parts (see figure 1): (1) the cathode sheath (which in its turn consists of the Aston dark space where the electrons leaving the cathode do not yet possess the energy required for gas molecule excitation, cathode glowing sheath where electrons already possess the energy for a direct or stepwise gas molecule excitation, and the cathode (Crookes) dark space where the intense processes of ionizing and exciting gas molecules via electron avalanches occur); (2) the bright negative glow (the voltage drop across it is small, and the ionization and excitation of gas 


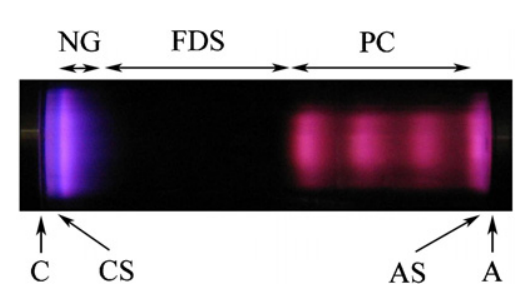

Figure 1. Photo of dc discharge, $L=400 \mathrm{~mm}, p=0.2$ Torr, $U=1200 \mathrm{~V}$. 'C'-cathode, 'CS'cathode sheath, 'NG' — negative glow, 'FDS' - Faraday dark space, 'PC'—positive column (with striations), 'AS' - anode sheath, 'A' - anode.

molecules are accomplished by a directed flow of electrons accelerated in the cathode sheath); (3) the Faraday dark space (across which the voltage drop from the negative glow side is small but then it grows approaching the anode, the disordered motion of electrons prevailing over its ordered motion); (4) the positive column (in which in the absence of striations the longitudinal field strength remains constant, and the potential grows linearly along the discharge axis approaching the anode); (5) the anode sheath (which in its turn consists of the anode dark space and the anode glow with intense ionization of gas molecules via electron impact and a considerable voltage drop). Consequently, the high voltage drop is located across the cathode sheath as well as the positive column and the anode sheath [10-12].

In short discharge tubes the positive column is absent, and the anode glow (and the anode voltage drop) appears only at gas pressure values to the right of the inflection point at the dc discharge breakdown curve [10,12]. At low pressure the discharge in a short tube consists only of the cathode sheath, the negative glow, and a portion of the Faraday dark space may also be observed. In the photo (see figure 2) the cathode sheath is between the cathode ' $\mathrm{C}$ ' and the boundary of the cathode sheath. To the right of the cathode sheath boundary we observe the negative glow spread up to the anode 'A'. Under these conditions the voltage drop across the electrodes is actually equal to the voltage drop across the cathode sheath $U$. The sheath thickness may be easily estimated visually because the boundary between the cathode sheath and the negative glow is usually clear cut. The current across the cathode sheath is due to positive ions whereas the electron component of the current is small. In the abnormal regime the discharge covers the cathode surface entirely; therefore, the current is $I=J \cdot S$, where $S$ is the cathode area.

The aim of this laboratory work is to decide which of the Child-Langmuir law versions (1)-(3) is the most appropriate for the description of the cathode sheath of the dc discharge with the help of simple measurements of the voltage drop across the electrodes, the current $I$ and the cathode sheath thickness $d$.

\section{Experimental conditions for performing the laboratory work}

To perform this laboratory work we need a conventional pre-vacuum (mechanical) pump. It is quite sufficient to employ such a pump because this work may be accomplished with low pressure air or nitrogen. Therefore, we need not use, say, a turbo-molecular pump to reduce limiting vacuum conditions in the discharge chamber.

We also need a source of dc voltage permitting a voltage drop to be created in the range 100-2000 V. This voltage drop is applied across the plane parallel electrodes placed inside a transparent cylindrical discharge tube (made of fused silica or glass). Electrode diameter may be, say, 3-10 cm. It is desirable that the electrodes almost fill the cross section of the discharge 


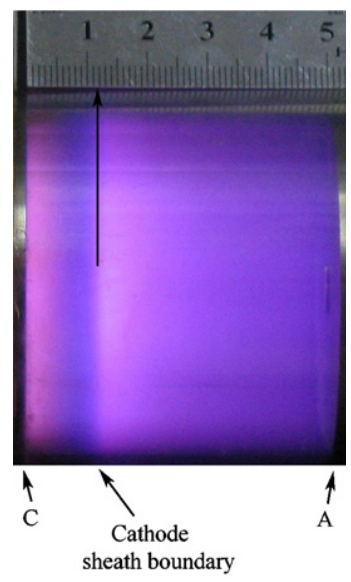

Figure 2. Photo of dc discharge, $L=50 \mathrm{~mm}, p=0.1$ Torr, $U=800 \mathrm{~V}$. 'C' —cathode, 'A'-anode.

tube. Otherwise the discharge may also burn inside the gap between the lateral surface of the electrode and the tube wall, and it would impede the evaluation of the cathode sheath thickness. When this gap is used for gas supplying or evacuating it does not have to exceed $1 \mathrm{~mm}$.

We attach a narrow strip of paper with millimetre scale outside the tube. Zero corresponds to the cathode surface. This scale permits the evaluation of the cathode sheath thickness. Otherwise we may attach a millimetre ruler outside the discharge tube. But in this case it is expedient to photograph the discharge and then to determine the cathode sheath thickness on the monitor screen.

The discharge chamber is filled with a gas under study (nitrogen, argon, air) which is then pumped out with the above-mentioned mechanical pump.

The experimental setup is equipped with a voltmeter (say, to $2000 \mathrm{~V}$ ), connected in parallel to the chamber electrodes. The same electrodes are fed with a dc voltage from a voltage source, and the electrode circuit is supplied with a milliammeter (up to $200 \mathrm{~mA}$ ) and a resistor $R \approx 10-300 \mathrm{k} \Omega$ to limit the discharge current and to impede the appearance of undesirable cathode spots.

Now let us determine the range of gas pressure $p$ suitable for performing the measurements. As was noted above, we need to create a dc discharge consisting of the cathode sheath and the negative glow with the optional presence of the Faraday dark space. The experiments are better performed near and to the left of the dc breakdown curve minimum for the available chamber (figure 3 ) constructed to scale $U_{\mathrm{br}}(p L)\left(U_{\mathrm{br}}\right.$ is the breakdown voltage and $L$ is the inter-electrode gap). The inter-electrode gap value is kept unchanged. Students at our university register this breakdown curve in the course of accomplishing one of the preceding undergraduate laboratory works. To this end they establish fixed values of the gas pressure and then increase the voltage slowly until the breakdown occurs. The inter-electrode gap value is kept unchanged.

The value of the product $(p L)_{\min }$ at the minimum depends on the electrode material and the ratio of the gap width to the tube radius $L / R_{t}$. But at $L / R_{t} \leqslant 1$ for a cathode of conventional materials (stainless steel, aluminium, copper, titanium) the minimum is located in air at $(p L)_{\min } \approx 0.55$ Torr $\mathrm{cm}$, in nitrogen at $(p L)_{\min } \approx 0.6$ Torr $\mathrm{cm}$ and in argon at $(p L)_{\text {min }} \approx 1.5$ Torr $\mathrm{cm}[12]$. Therefore, the pressure range $p \leqslant \frac{(p L)_{\min }}{L}$ is to be preferred. At higher pressure values the voltage drop across the Faraday dark space increases, and on approaching the inflection point at the dc breakdown curve (at $(p L)_{\text {inf }}=e(p L)_{\min }, e$ is the base of natural logarithms) a 


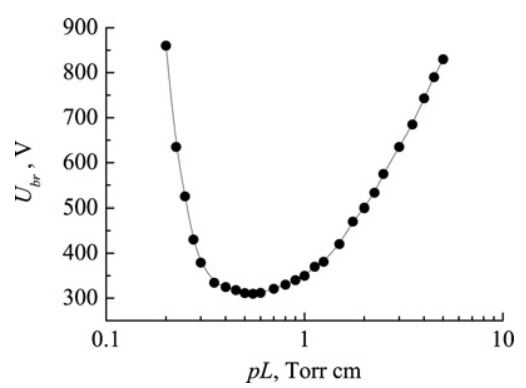

Figure 3. The dc breakdown curve in nitrogen, $L=50 \mathrm{~mm}$.

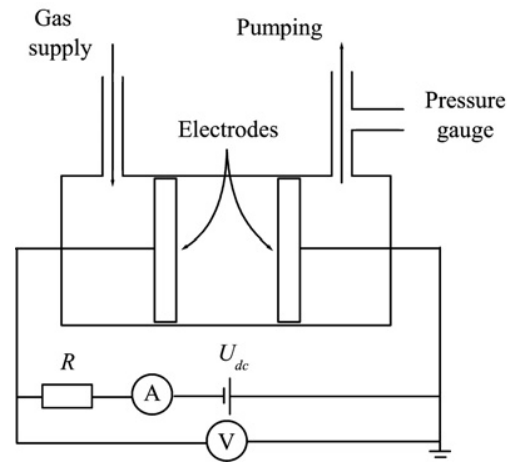

Figure 4. Scheme of the experimental device.

glow appears near the anode with a voltage drop around 10-20 V [7, 8]. This anode voltage drop would cause an error in registering the voltage drop across the cathode sheath. Therefore, we suggest limiting the pressure range to the values near to and to the left of the dc breakdown curve minimum.

However, the gas pressure also should not be too low because otherwise the collisiondominated Child-Langmuir law (2), (3) would cease to be valid. Therefore, we choose the pressure range for performing the laboratory work as

$$
0.03 \text { Torr } \leqslant p \leqslant \frac{(p L)_{\min }}{L} .
$$

The optimum inter-electrode gap width amounts to $L \approx 2-5 \mathrm{~cm}$, because with larger gap widths $L$, the dc breakdown curve minimum is shifted to lower gas pressure values and it approaches the left limit (5).

In the device we employed (figure 4) the diameter of stainless steel electrodes was $55 \mathrm{~mm}$. The gas was supplied and evacuated through the grounded electrode. The inter-electrode gap $L$ was $50 \mathrm{~mm}$.

\section{Performing the work}

After the students are acquainted with the discharge chamber, registering devices, a gas supply system and a pumping system and experimental conditions, they put on the setup and get the limiting vacuum condition. Then they supply the gas under study (e.g., nitrogen) to the 


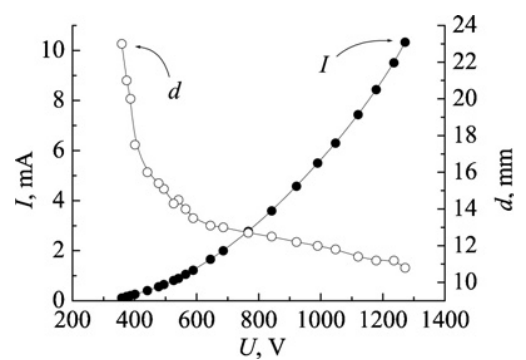

Figure 5. Discharge current $I$ and cathode sheath thickness $d$ against voltage drop $U$ across the electrodes for the nitrogen pressure of 0.1 Torr and inter-electrode distance of $50 \mathrm{~mm}$.

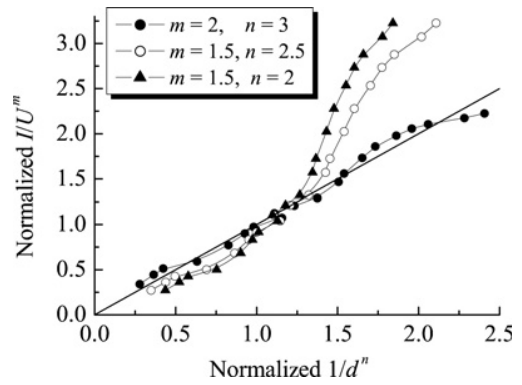

Figure 6. Normalized $I / U^{m}$ as a function of normalized $1 / d^{n}$ for different values of $m$ and $n$ for the nitrogen pressure of 0.1 Torr and inter-electrode distance of $50 \mathrm{~mm}$.

pressure 0.05 Torr. After that they use the vacuum valve to increase the pressure to 0.1 Torr. At this gas pressure and inter-electrode gap values the product $p L$ is somewhat less than $(p L)_{\min }$ at the dc breakdown curve in nitrogen (figure 3 ).

Then they put on the dc voltage source and establish fixed values of the voltage $U$, registering the discharge current $I$ and the cathode sheath thickness $d$ (figure 5). The measurements are performed for 10-20 values of the dc voltage $U$ in the range 300$2000 \mathrm{~V}$. The data collected in this way are shown in figure 5. This figure demonstrates that with increasing dc voltage the discharge current increases whereas the cathode sheath thickness decreases. It also follows from figure 5 that current $I$ is a strong function of voltage $U$, and the cathode sheath thickness $d$ first experiences a fast decrease with $U$ growing, but at voltage values above $600 \mathrm{~V}$ its dependence on $U$ is weak.

After that the students calculate the parameters $I / U^{m}$ and $1 / d^{n}$, using the data from figure 5, for each pair of $m$ and $n(m=1.5$ and $n=2, m=1.5$ and $n=2.5, m=2$ and $n=3$ ). Then these parameters have to be normalized. To this end we divide each value by the average value for this parameter. Figure 6 presents the normalized parameters $I / U^{m}$ and $1 / d^{n}$ and it makes evident that for $m=2$ and $n=3$ the dependence closest to the linear one is observed. It means that under conditions of the experiment performed version (3) of the collision-dominated Child-Langmuir law is applicable in which the ion mobility $\mu_{i}$ is independent of velocity. 


\section{Conclusion}

Thus we propose in this paper how the students may validate the collision-dominated ChildLangmuir law in the course of measuring the characteristics of the dc discharge cathode sheath in a simple laboratory setup. To accomplish this task they are to perform simple measurements of the voltage drop across the electrodes, the discharge current through the device and the cathode sheath thickness.

\section{Acknowledgment}

We are indebted to the referee for his invaluable remarks and suggestions which helped to improve the content and outline of the presented material.

\section{References}

[1] Schaaf P 2005 Das Physikalische Praktikum Fuer Studentinnen und Studenten der Physik Handbuch fuer das Praktikum (ab SS2005) Universitaetsdrucke Goettingen S.153

[2] Child C D 1911 Phys. Rev. 32492

[3] Langmuir I 1913 Phys. Rev. 2450

[4] Langmuir I 1923 Phys. Rev. 21419

[5] Azooz A A 2007 Eur. J. Phys. 28635

[6] Langmuir I 1929 Phys. Rev. 33954

[7] Raizer Y P 1991 Gas Discharge Physics (Berlin: Springer)

[8] Lieberman M A and Lichtenberg A J 1994 Principles of Plasma Discharges and Materials Processing (New York: Wiley)

[9] Francis G 1956 The glow discharge at low pressure Handbuch der Physik vol XXII ed S Flugge (Berlin: Springer) p 54

[10] Lisovskiy V A and Yakovin S D 2000 Tech. Phys. Lett. 26891

[11] Lisovskiy V A and Yakovin S D 2000 Plasma Phys. Rep. 261066

[12] Lisovskiy V A, Yakovin S D and Yegorenkov V D 2000 J. Phys. D: Appl. Phys. 332722 